\title{
Surface-Stress Induced Embrittlement of Metals Supplementary Information
}

\author{
Anirudh Udupa ${ }^{* \dagger 1}$, Tatsuya Sugihara ${ }^{\dagger 2}$, Koushik Viswanathan $^{3}$, Ronald M. \\ LATANision $^{4}$, AND SRINIVASAN CHANDRASEKAR ${ }^{1}$ \\ ${ }^{1}$ Center for Materials Processing and Tribology, Purdue University, West Lafayette, IN, USA - \\ 47906 \\ ${ }^{2}$ Department of Mechanical Engineering, Osaka University, Suita, Osaka 565-0871, Japan \\ ${ }^{3}$ Department of Mechanical Engineering, Indian Institute of Science, Bangalore 560012, India \\ ${ }^{4}$ Materials Science and Engineering, MIT, Cambridge, MA, USA - 02139, and Exponent Inc., \\ Natick, MA, USA - 01760
}

October 28, 2021

*Corresponding author. Email: audupa@purdue.edu

${ }^{\dagger} \mathrm{AU}$ and TS contributed equally to this paper. 


\section{Experimental details}

The experimental framework for applying the simple shear deformation used a model 2D cutting configuration, in which a hard wedge (tool) was moved relative to a workpiece at velocity $V_{0}$ to remove a strip of material (chip) (see Fig. 3a). This causes a peeled layer (chip) to form by simple shear imposed over a narrow (red) deformation zone [?], as shown in the figure. The wedge face was fixed normal to $V_{0}$, making an angle $\alpha$ with the vertical (rake angle). The workpiece was constrained on one side by an optically transparent glass block, to ensure conditions of plane-strain (2D) deformation. The depth of material peeled (undeformed chip thickness, $h_{0}$ ) was set nominally at $50 \mu \mathrm{m}$; the exact $h_{0}$ was obtained by direct measurement. A nominal sliding velocity $V_{0}=5$ $\mathrm{mm} / \mathrm{s}$ was used in all of the experiments; at this small velocity, the deformation is essentially quasi-static and temperature effects are negligible.

The material (plastic flow) in the workpiece was observed in situ at high resolution using an optical microscope coupled to a high-speed complementary metal-oxide semiconductor (CMOS) camera (Photron WX-100). The deformation zone was illuminated by a 120-W halogen lamp. A framing rate of 500 frames per second was used. Under these imaging conditions, the spatial resolution was $1.1 \mu \mathrm{m}$ per pixel. The camera output was high-speed image sequences of the material flow in and around the deformation zone. The high-speed image sequences were analyzed using methods of Digital Image Correlation (DIC) to obtain accurate interframe material displacements. From the displacement fields, a record of material velocity, and strain/strain-rate field histories was derived, enabling quantitative characterization of material deformation. The flow was also visualized using streaklines, and strain fields. A streakline is defined as the locus of a fixed set of material points, as they are convected during material deformation.

The workpiece material was commercially pure aluminum (Al 1100, 99.0\%, McMaster-Carr) in the form of a rectangular block of dimension $75 \mathrm{~mm}$ (length) $\times 25 \mathrm{~mm} \times 3 \mathrm{~mm}$ (width into the plane, Fig. 3a). It was annealed in a furnace at $550{ }^{\circ} \mathrm{C}$ for $4 \mathrm{~h}$ and oven cooled to room temperature. The initial hardness was $23 \mathrm{HV}$ (annealed) and the grain size was $\sim 200 \mu \mathrm{m}$. The hard wedge was made of WC-Co cemented carbide. The deformation force (parallel to $V_{0}$ ) was measured using a piezoelectric dynamometer (Kistler 9257B), concurrent with the high-speed imaging of the 
deformation.

\subsection{Preparation of organic monolayers}

We prepared Al, with various organic monolayers on its surface, using well-documented procedures for Self-Assembled Monolayer (SAM) films [1]. In all of the experiments, we have used the annealed aluminum (with its native surface oxide layer) as the substrate and various organic molecules as the monolayer adsorbate. Specifically we used five Sil-X-CH 3 molecules — trichloro(propyl)silane(Sil3- $\left.\mathrm{CH}_{3}\right)$, trichloro(hexyl)silane (Sil-6- $\left.\mathrm{CH}_{3}\right)$, trichloro(octyl)silane( $\left.\mathrm{Sil}-8 \mathrm{CH}_{3}\right)$, trichloro(decyl)silane $\left(\mathrm{Sil}-10 \mathrm{CH}_{3}\right)$ and trichloro(hexadecyl)silane $\left(\mathrm{Sil}-16-\mathrm{CH}_{3}\right)$. In addition we deposited several other short and long-chain monolayers: one $\mathrm{CA}-\mathrm{X}-\mathrm{CH}_{3}$ molecule, viz., stearic acid $\left(\mathrm{CA}-16-\mathrm{CH}_{3}\right)$; and monolayers of Tetramethyl Orthosilicate (Sil-0-OH), (3-Mercaptopropyl)trimethoxysilane (Sil-3$\mathrm{SH})$, Trimethoxy $\left(1 \mathrm{H}, 1 \mathrm{H}, 2 \mathrm{H}, 2 \mathrm{H}\right.$-heptadecafluorodecyl)silane (Sil-10-CF $\left.\mathrm{CF}_{3}\right)$ and (11-Azidoundecyl)trimethoxysile (Sil-11-N ${ }_{3}$. The general procedure used to deposit the organic monolayers was as follows.

The Al workpiece surface was subjected to an initial plasma irradiation treatment (He gas, 100 $\mathrm{Pa}, 200 \mathrm{~W}, 120 \mathrm{~s})$ to increase the density of -OH groups on the surface. This treatment is necessary for the native aluminum oxide layer to form a strong bond with the organic molecules. Next, a 1 $\mathrm{mM}$ solution of one of the aforementioned organic molecules in ethanol was prepared. In addition to the organic molecule, dibutylin dilaurate was used as a catalyst and N-(1,3-dimethylbutylidene)3-triethoxysilyl-1-propanamine was used as a promoter, each mixed into the ethanol solution at a concentration of $1 \mathrm{mM}$ each [2]. The Al workpiece was then immersed in this solution for 1 hour, followed by air-drying at room temperature $(\mathrm{T} \sim 300 \mathrm{~K})$ for 12 hours. Finally, the workpiece was again cleaned ultrasonically in an ethanol bath for $5 \mathrm{~min}$, and in pure water for $5 \mathrm{~min}$, to complete the sample preparation. The catalyst and promoter were not used in the case of $\mathrm{CA}-16-\mathrm{CH}_{3}$.

\subsection{Measurement of organic monolayer thickness}

The thickness of the organic monolayers was measured using spectroscopic ellipsometry (Horiba, Uvisel Lt NIR-NNG). The angle of incidence was $70^{\circ}$ and the spot size $2 \mathrm{~mm} \times 6 \mathrm{~mm}$. The thickness

of the native oxide layer was measured to be equal to $49.6 \AA$. The refractive index and extinction 
coefficient of the substrate were obtained by point-by-point calculation process [?]. In order to accurately measure the thickness of the organic monolayer, it is important to deposit it on an atomically smooth substrate. Else, the roughness of the substrate will create variations in the measurements that are typically greater than the monolayer thickness, thereby making readings difficult to interpret. Hence, a smooth aluminum surface was prepared by sputtering $300 \mathrm{~nm}$ of $\mathrm{Al}$ on a 2" Si-wafer. This smooth substrate was then subjected to the same treatment as the aluminum workpiece described above to deposit the monolayers. The results of the thickness measurements are shown in Figure 2. The ratio of this thickness to the actual length of the molecule gives the cosine of the angle that the molecule makes with the surface normal. This angle was found to be $32 \pm 4^{\circ}$, similar to other reported values $[3]$.

\section{Simulation details}

We performed two sets of molecular dynamics simulations: i) to estimate surface energy and surface stress for various organic adsorbates on the $\mathrm{Al}_{2} \mathrm{O}_{3}$ substrate and ii) to study the effect of an adsorbate induced surface stress on material deformation response. The details of the simulation procedure are given below.

\subsection{Simulations to estimate surface stress, $f$, and surface/interface energy, $\gamma$}

As mentioned in the main article, the simulation configuration is that of an organic monolayer adsorbate on one-side of an $\mathrm{Al}_{2} \mathrm{O}_{3}$ (substrate) beam (free at both ends). The organic adsorbates that we simulated are all of the $\mathrm{CA}-\mathrm{X}-\mathrm{CH}_{3}$ variety as the interatomic potentials are readily availble for these. Whereas for the silane molecules, the potentials are not freely available. We used the OPLS interatomic potential [4] to simulate the organic adsorbates and the Vashishta potential [5] for $\mathrm{Al}_{2} \mathrm{O}_{3}$. The change in curvature of the $\mathrm{Al}_{2} \mathrm{O}_{3}$ beam resulting from the application of the monolayer films was calculated. This was then used in conjunction with Stoney's equation to estimate $f$. 
The adsorbate molecules were arranged in $2 \times 2 R 0^{\circ}$ on the cantilever corresponding to an intermolecular spacing of $5.3 \AA$. The $\mathrm{Al}_{2} \mathrm{O}_{3}$ substrate was simulated using the Vashishta potential [5] and the CA molecules were simulated with the OPLS all-atom potential [4]. Previous FTIR studies have found that the CA molecule dissociates by losing the proton from the -COOH head group before bonding with the substrate [1,6]. Specifically, the -COO- group forms a bond with the aluminum atom in the alumina substrate. The partial charges on the bonding atoms (oxygen from the $-\mathrm{COO}^{-}$head group and the $\mathrm{Al}$ from the $\mathrm{Al}_{2} \mathrm{O}_{3}$ substrate) are assumed to be the same as in $\alpha-\mathrm{Al}_{2} \mathrm{O}_{3}[5]$. When adsorption is complete, a series of adjacent electric dipoles are pinned to the surface, all repelling each other through Coulombic interaction [7]. This repulsion typically results in a compressive surface stress.

The size of the simulated $\mathrm{Al}_{2} \mathrm{O}_{3}$ beam was $25 \mathrm{~nm} \times 5 \mathrm{~nm} \times 4 \mathrm{~nm}$. We repeated the simulations for two adsorbates $\left(\mathrm{CA}-6-\mathrm{CH}_{3}\right.$ and $\left.\mathrm{CA}-16-\mathrm{CH}_{3}\right)$ with a larger beam $(50 \mathrm{~nm} \times 5 \mathrm{~nm} \times 8 \mathrm{~nm})$ to observe if there was any change in the predicted $f$. The predicted $f$ for these two cases was, -0.98 $\mathrm{N} / \mathrm{m}$ and $+1.96 \mathrm{~N} / \mathrm{m}$, the same as with the smaller beam, indicating that the $f$ estimates are independent of beam dimension.

The energy of the $\mathrm{Al}_{2} \mathrm{O}_{3}$-adsorbate interface, $\gamma$, was calculated as the difference in the energy of the system prior to and after adsorption. More specifically, $\gamma=E_{\mathrm{Al}_{2} \mathrm{O}_{3}}+E_{\text {adsorbate }}-E_{\mathrm{Al}_{2} \mathrm{O}_{3}+\text { adsorbate }}$, where $E$ denotes the energy of the system in the subscript. The change in $\gamma$ with $l_{c}$ is shown in Fig. 1c with the black curve.

In order to calculate the surface energy of $\mathrm{Al}_{2} \mathrm{O}_{3}$ itself, we calculated the difference of the energies of two simulations with and without periodic boundary conditions. Specifically, we performed one set of simulations with periodic boundary conditions in all directions and calculated the equilibirum energy $\left(E_{\mathrm{Al}_{2} \mathrm{O}_{3}}^{\mathrm{pbc}}\right)$. We then performed another set of simulations with periodic boundary conditions relaxed only in the Z-direction $\left(E_{\mathrm{Al}_{2} \mathrm{O}_{3}}^{\text {free }}\right)$. We then estimated the $\mathrm{Al}_{2} \mathrm{O}_{3}$ surface energy (also denoted as $\gamma$ ) as $\gamma=0.5 *\left(E_{A l_{2} O_{3}}^{f r e e}-E_{A l_{2} O_{3}}^{p b c}\right)$. This was calculated as equal to $1.85 \mathrm{~J} / \mathrm{m}^{2}$, in agreement with published literature values [?]. 


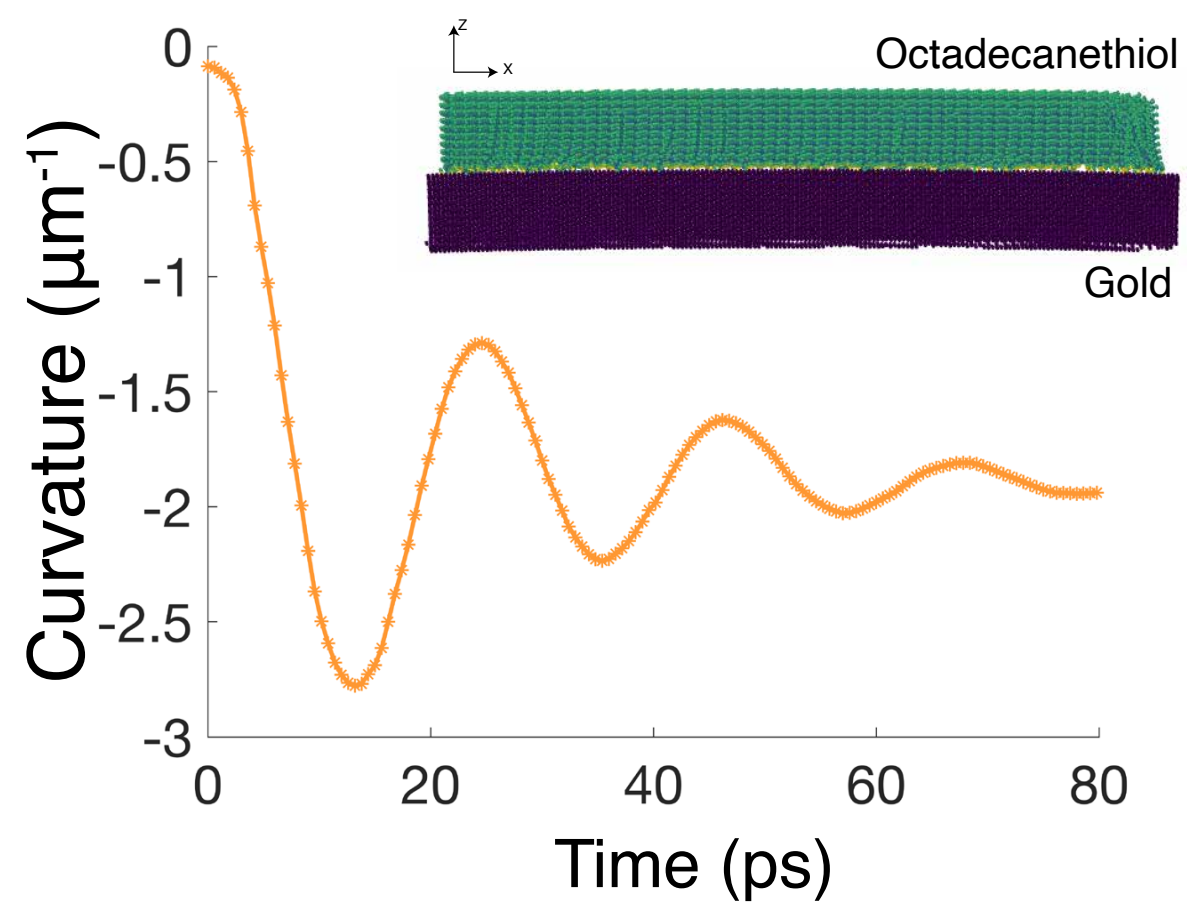

Figure 1: Simulation of adsorption of octadecanethiol on gold substrate. Graph shows variation in curvature of the gold-beam as a function of time. Negative curvature (adsorbate on convex side) implies compressive surface stress. Inset shows snapshot of the equilibrium configuration of the gold-octadecanethiol system.

\subsection{Validation with gold-alkanethiol system}

In addition to the simulations with the $\mathrm{Al}_{2} \mathrm{O}_{3}-\mathrm{CA}-\mathrm{X}-\mathrm{CH}_{3}$ system for estimating $f$, we also simulated adsorption of octadecanethiol on a gold substrate (Fig. S1(inset)) using a similar methodology. This system was selected as a validator for the simulations because it has been wellcharacterized and studied extensively $[7-10]$. The gold atoms were simulated using a Lennard-Jones potential and the octadecanethiol molecules once again using the OPLS interatomic potential. The $\mathrm{X}-\mathrm{Y}$ plane corresponds to the $\langle 111\rangle$ plane of the gold atoms and the alkanethiol molecules are arranged in a $\sqrt{3} \times \sqrt{3}$ R30 lattice on the beam surface. Charges to the gold and sulfur atoms involved in the bonding were assigned according to XPS measurements [11]. The variation in curvature of the gold-beam is showing in Fig. S1. The $f$ corresponding to the equilibrium curvature was calculated to be $-0.6 \mathrm{~N} / \mathrm{m}$ within the range of previous measurements and calculations $7,8,10]$. This result serves to validate the stress simulation procedure. 


\subsection{Simulations to study effect of surface stress on metal deformation response}

In order to study ductile versus brittle behavior of crystals, a key feature of sinuous-to-segmented flow transition underlying the OME phenomenon, we use the following framework inspired by the Rice-Thomson model [12]. The approach is to explore the behavior of an atomically-sharp notch tip in a material that is loaded remotely in tension. When the load reaches a value equal to the Griffith stress, depending on the material properties, the notch will do one of the following two things, 1) allow a crack to propagate into the material, thus extending the length of the crack (brittle) or 2) emit dislocations and get blunted in the process (ductile). Crystals for which dislocation emission is spontaneous can be expected to be ductile and others wherein there exists a large energy barrier for this emission can be expected to be good candidates for brittle cleavage. We extend this framework to probe how the behavior of a crystal changes in the presence of a surface-stress at the notch-tip induced by an adsorbate. With this aim we perform a second set of molecular dynamics simulations of a tensile test on a notched (atomically sharp) specimen. We studied the notch-tip behavior with and without adsorbate atoms. The surface stress exerted by the adsorbate atoms was varied from $f=-3$ to $+3 \mathrm{~N} / \mathrm{m}$ (see details below).

In these second set of simulations, an orthorhombic aluminum crystal $\left(L_{x} \times L_{y} \times L_{z}=40 \mathrm{~nm} \times\right.$ $5 \mathrm{~nm} \times 40 \mathrm{~nm})$ containing a notch is subjected to remote tensile loading. The notch was $\sim 2 \mathrm{~nm}$ in length and $\sim 0.8 \mathrm{~nm}$ in width and lies parallel to the X-direction $(\langle 100\rangle)$. The model material, aluminum, simulated using the Embedded Atom Method (EAM) interatomic potential. Periodic boundary conditions $(\mathrm{PBC})$ are imposed in the Y-direction to simulate plane-strain conditions. $T=300 \mathrm{~K}$, an NVE ensemble with a Langevin thermostat and velocity-Verlet algorithm with integration time steps of $1 \mathrm{fs}$ were used in all the simulations. The remote tensile loading was simulated by moving the top-row of atoms in the Z-direction, maintaining a strain-rate of $10^{6} \mathrm{~s}^{-1}$, while holding the bottom row of atoms fixed stationary.

The dimulations corresponding to Figs. 4D, $\mathbf{4 E}$ had adsorbate atoms at the notch-tip. The adsorbate atoms were modeled as Lennard-Jones (LJ) solid. This facilitates minimization of the parameters that the adsorbate brings to the simulation, such as charge of atoms, polarity etc., 
thereby making the results easy to interpret. The adsorbate induced surface-stress at the notchtip was controlled by changing the equilibrium distance between the LJ adsorbate molecules. The lattice-mismatch between the LJ atoms and the aluminum atoms can be modified to control the magnitude and sign of the surface stress. In the actual experiments, the SAM molecules induce surface stress on the metal workpiece because of van-der Waals interactions between the long chain molecules, not because of a lattice mismatch.

\section{Captions for Movies}

Movie 1: A bare annealed $\mathrm{Al}$ workpiece deforms via sinuous flow to form a thick chip with wavy streaklines and heterogeneous strains.

Movie 2: An annealed Al workpiece with stearic acid monolayer (long-chain molecule) cracks initiate repeatedly from the surface and propagate inwards. These cracks arrest the development of sinuous flow, thus resulting in a relatively thin chip with segments separated by cracks. The deformation force is $85 \%$ smaller with the SAM layer as compared to without the layer.

Movie 3: Atomistic simulation of a tensile test of an atomically sharp notched crystal of aluminum at room temperature. Simulations predict that the notch repeatedly emits dislocations characteristic of a ductile material.

Movie 4: Atomistic simulation of a tensile test of an atomically sharp notched crystal of aluminum with an adsorbate that induced a compressive surface stress of $-0.6 \mathrm{~N} / \mathrm{m}$. Simulations predict that the notch repeatedly emits dislocations characteristic of a ductile material.

Movie 5: Atomistic simulation of a tensile test of an atomically sharp notched crystal of aluminum with an adsorbate that induced a tensile surface stress of $+0.4 \mathrm{~N} / \mathrm{m}$. Simulations predict that the notch cleaves and a crack propagates typical of a brittle material.

\section{References}

[1] Ulman A. Formation and structure of self-assembled monolayers. Chemical Reviews. 1996;96(4):1533-1554. 
[2] Kim YJ, Lee KH, Sano H, Han J, Ichii T, Murase K, et al. Surface chemical conversion of organosilane self-assembled monolayers with active oxygen species generated by vacuum ultraviolet irradiation of atmospheric oxygen molecules. Japanese Journal of Applied Physics. 2008;47(1R):307.

[3] Genzer J, Efimenko K, Fischer DA. Molecular orientation and grafting density in semifluorinated self-assembled monolayers of mono-, di-, and trichloro silanes on silica substrates. Langmuir. 2002;18(24):9307-9311.

[4] Jorgensen WL, Tirado-Rives J. Potential energy functions for atomic-level simulations of water and organic and biomolecular systems. Proceedings of the National Academy of Sciences. 2005;102(19):6665-6670.

[5] Vashishta P, Kalia RK, Nakano A, Rino JP. Interaction potentials for alumina and molecular dynamics simulations of amorphous and liquid alumina. Journal of applied physics. 2008;103(8):083504.

[6] Tao YT. Structural comparison of self-assembled monolayers of n-alkanoic acids on the surfaces of silver, copper, and aluminum. Journal of the American Chemical Society. 1993;115(10):4350-4358.

[7] Godin M, Tabard-Cossa V, Miyahara Y, Monga T, Williams P, Beaulieu L, et al. Cantileverbased sensing: the origin of surface stress and optimization strategies. Nanotechnology. 2010;21(7):075501.

[8] Berger R, Delamarche E, Lang HP, Gerber C, Gimzewski JK, Meyer E, et al. Surface stress in the self-assembly of alkanethiols on gold. Science. 1997;276(5321):2021-2024.

[9] Godin M, Williams P, Tabard-Cossa V, Laroche O, Beaulieu L, Lennox R, et al. Surface stress, kinetics, and structure of alkanethiol self-assembled monolayers. Langmuir. 2004;20(17):70907096. 
[10] Desikan R, Lee I, Thundat T. Effect of nanometer surface morphology on surface stress and adsorption kinetics of alkanethiol self-assembled monolayers. Ultramicroscopy. 2006;106(89):795-799.

[11] Bourg MC, Badia A, Lennox RB. Gold- sulfur bonding in 2D and 3D self-assembled monolayers: XPS characterization. The Journal of Physical Chemistry B. 2000;104(28):6562-6567.

[12] Rice JR, Thomson R. Ductile versus brittle behaviour of crystals. The Philosophical Magazine: A Journal of Theoretical Experimental and Applied Physics. 1974;29(1):73-97. 